\title{
Lessons Learned from the 2017 Kermanshah Earthquake: The Presence of Celebrities in the Field
}

\author{
Alireza Mashallahi ${ }^{1}$, Milad Ahmadi Marzaleh ${ }^{2}$ and Abbas Ostadtaghizadeh ${ }^{3,{ }^{*}}$ \\ ${ }^{1}$ School of Public Health, Tehran University of Medical Sciences, Tehran, Iran \\ ${ }^{2}$ Student Research Committee, Department of Health in Disasters and Emergencies, Health Human Resources Research Center, School of Management and Medical \\ Informatics, Shiraz University of Medical Sciences, Shiraz, Iran \\ ${ }^{3}$ Department of Disaster Public Health, School of Public Health, Tehran University of Medical Sciences, Tehran, Iran \\ "Corresponding author: Ph.D. of Health in Disasters and Emergencies, Department of Disaster Public Health, School of Public Health, Tehran University of Medical Sciences, \\ Tehran, Iran. Email: ostadtaghizadeh@gmail.com
}

Received 2019 February 27; Accepted 2019 March 05

Keywords: Aid, Altruism, Celebrity, Earthquakes, Humanitarian, Non-Governmental, Organizations

\section{Dear Editor,}

Ezgeleh is a city in Kermanshah province, in western Iran. According to the census 2016, the city population was 1502 individuals. At 21:48 PM on the 12th of November 2017, an earthquake with a magnitude of 7.3-Richter Scale struck the city at the depth of 11-kilometer. The epicenter was in Ezgeleh, a district of Salase-e-Babajani county near the city of Sar-Pol-e-Zahab, both of which sustained the highest damage. Based on the last statistics by the forensic medicine association, the number of the lives lost in Kermanshah earthquake was 620 individuals. Additionally, 9388 individuals were injured, and about 70000 individuals became homeless $(1,2)$.

One of the main weaknesses in the management practices in this earthquake was the absence of an NGOs in the region to direct and guide the people-driven aids. However, due to lack of trust in the government agencies and nonexistence of NGOs, celebrities attempted to help people by taking the initiative. Even though celebrities enter the arena in the course of such events, usually an organization takes the main responsibility and the celebrities usually play a role of an advertiser or organizer, and not many celebrities around the globe announce their own personal account number for helping people. However, the issue here is that they could have organized and managed the people's needs better, because the relief responsibility and/or even the transferring of people's financial and non-financial aids demands a great deal of responsibility. Amongst the structural shortages of celebrities' movements, the following factors can be pointed out:
- Paying no attention to the social abnormalities and psychological problems

- Having no codified program for short- and long-term shelters for the victims

- Absence of standards and regulations for celebrities to in provide assistance and lack of infrastructure for their cooperation with other organizations

- Absence of efficient and expert teams in various areas

- Ignoring the cultural structure of the earthquakestricken region

- Mere emphasis by some celebrities on collecting financial aids, but not on the no psychological and emotional supports

Due to the zealous presence of celebrities in the field, a sense of social responsibility was observed which was taken as a good omen, regarding their presence in executive activities, which they had no prior experience, as well as announcing their own personal bank accounts that was somehow accompanied by a sort of social trust, the society was witnessing a national excitement and fervor. In the short run, the celebrities moved toward social solidarity and responsibility, but its discontinuation brought about substantial problems like mistrust in the agencies, and this can per- se exert deeper effect on the future problems.

At the end, having a great deal of effect on people, celebrities can keep the social solidarity movement going in an organized fashion, but their interventions should be monitored, evaluated and organized by the government agencies such as the Red Crescent. 


\section{Footnotes}

Conflict of Interests: The authors do not have any conflict of interests to declare.

Funding/Support: It is not declared by the authors.

\section{References}

1. Ostadtaghizadeh A, Khaleghy Rad M, Aghababaeian H, Zare M, Kamranzad F. Earthquake in western Iran: Renovation kills. PLoS Currents. 2018. doi: 10.1371/currents.dis.ac10620db4a0c944e605c4c226a75f64.

2. Ahmadi A, Bazargan-Hejazi S. 2017 Kermanshah earthquake; lessons learned. J Inj Violence Res. 2018;10(1):1-2. doi: 10.5249/jivr.v10i1.1049. [PubMed: 29374766]. [PubMed Central: PMC5801606]. 